\title{
PHILOSOPHY OF PHYSICS
}

Tim Maudlin

Original Scientific Paper

NYU

UDK 530.145:111

\section{THE METAPHYSICS OF QUANTUM THEORY}

Why does philosophy of physics exist? Physics has been historically important to philosophers, from Thales and Democritus and Empedocles through Plato and Aristotle, Descartes and Leibniz and Kant. All of these philosophers engaged in speculation about the physical world, and that speculation informed their philosophical views. But once physics became a specialized subject of its own the relation between physics and philosophy was bound to change. Physicists with no philosophical training or interests could advance physical theory. Philosophers could call on physics if necessary, but would have little to contribute to that field per se. In particular, philosophers interested in physical ontology could defer to the most recent physical theories. On this model, the philosopher is the junior partner, learning at the feet of the physicist about the physical world.

This account of the relationship between physics and philosophy might have been accurate. But in order for things to work this way, the physicist would have to supply clear answers to the philosopher's queries. What the philosopher wants to know, above all, is the ontology of the best physical theories, what those theories postulate as existing. And it is precisely here that the physicist sometimes fails to provide answers. Hence the need for the philosopher to clarify the situation.

A crucial example is provided by quantum theory. The physicist has a mathematical apparatus, and knows how to use it to make predictions. But a mathematical apparatus alone does not specify an ontology. It is a further step to explicate exactly how the mathematical objects are being used as representations of physical reality. In quantum theory, there is not even agreement about what it is a representation of.

Let's consider how the quantum physicist goes about modeling a concrete situation. There is a target system of interest, which is called a system of $\mathrm{N}$ particles. Already we are in contested territory. One should not presume, just because it is called an "N-particle system" that the physics is postulating that $\mathrm{N}$ particles, i.e. $\mathrm{N}$ small or pointlike items with definite positions at all times, exist. Indeed, most physicists will deny that there really are $\mathrm{N}$ particles in an "N-particle system". Why then the terminology? 
The talk of $\mathrm{N}$ particles arises as part of the construction of the mathematical apparatus. The physical state of a classical N-particle system is represented by a point in an N-particle configuration space. This is an abstract space of $3 \mathrm{~N}$ dimensions. Each point in the configuration space represents a possible configuration of the system, i.e., represents a set of $\mathrm{N}$ locations in physical space. The configuration space is, in essence, $\mathrm{N}$ copies of physical space, one copy for each particle. Classical configuration space does not represent a real physical space but is rather a convenient mathematical representation of a multiplicity of particles in real physical space. It is this $3 \mathrm{~N}$-dimensional mathematical space that the quantum physicist wants to employ in representing an "N-particle system".

But the $3 \mathrm{~N}$-dimensional space is not used as a configuration space: it is rather the space over which the wavefunction of the system is defined. While classical physics represents the state of a system by a point in configuration space, quantum theory uses instead a complex-valued function on the whole space. The wavefunction is supplied with a dynamics, a law of motion. In nonrelativistic quantum mechanics this is Schrodinger's equation.

At this point we can raise a central issue: what, if anything, does the wavefunction ascribed to a system represent? Does it represent anything physically real? If so, does it represent everything physically real in the system? If not, what else is there?

And we can be more precise: if the wavefunction represents something physically real, how does it do so? Which mathematical degrees of freedom in the representation correspond to physical degrees of freedom in the system? Or, to put it another way, when do two mathematically distinct wavefunctions represent the same physical situation?

Without answers to these questions the mathematical formalism alone cannot address the philosopher's ques tions.

Let's consider some of the proposed answers to these questions. The most basic question is whether the wavefunction represents any physical feature of a system at all. A negative response is offered by the quantum Bayesians (cf. Fuchs, Mermin and Schack 2014, 749). They hold that the wavefunction represents only the information some agent has about a system, and the Schrodinger equation specifies how that knowledge does-or ought to-evolve in time. So on this view quantum theory provides little or no insight into the objective physical situation.

Quantum Bayesianism disappoints one's hopes for insight into physical reality, but may seem to provide an escape for those disturbed by quantum theory. In particular, the quantum Bayesian seems to have a simple and satisfying understanding of the so-called collapse of the wavefunction: it is nothing but conditionalization on receipt of new information. If the wavefunction represents information then gaining new information would naturally cause it to suddenly change.

Do we have good reason to reject quantum Bayesianism and take the wavefunction more seriously as a representation of some feature of the system itself? A direct reason is provided by the iconic quantum phenomenon: the 
two-slit experiment. Particles shot one by one through a pair of open slits show interference bands. The experiment demonstrates that the system is physically sensitive to the state of both slits, so something must interact with both. The spreading of the wavefunction in space is not merely a matter of our becoming more uncertain where the particle is, but is rather a matter of some real physical item spreading enough to interact with both slits.

What's more, the wavefunction does not have the right form to directly represent a state of knowledge. A state of information would be represented by a probability measure over a set of propositions, but the wavefunction is not a probability measure. It is rather a probability amplitude: one needs to take the absolute square to get a probability measure. It is only because it is an amplitude that it can give rise to interference phenomena such as the two-slit experiment. So the wavefunction is not suited mathematically to represent information, and the attempt to interpret it as epistemic falls afoul of the very phenomena one wants to explain.

We are led to the conclusion that the wavefunction represents something physical. This still leaves open both whether it represents everything in the system and exactly how it represents the physical situation.

The view that the wavefunction represents everything, together with universal Schrodinger evolution, yields a Many Worlds theory. The Many Worlds character follows from the Schrodinger evolution. In a standard Schrodinger cat situation, for example, there is one experimental condition that will yield a live cat and another that will yield a dead cat. Let's call the resulting wavefunctions in these two cases $\mid$ alive $>$ and $\mid \mathrm{dead}>$. At this point we assume that the physical condition represented by the former contains a live cat and the physical condition represented by the latter a dead cat. We create a Schrodinger cat situation by. e.g., making the cat's state contingent on whether an electron is deflected up or down for a spin measurement in the $\mathrm{z}$-direction. If we feed an $\mathrm{x}$-spin up electron into the apparatus the linearity of Schrodinger evolution guarantees that the final state will be $1 / \sqrt{ } 2 \mid$ alive $>+1 / \sqrt{ } 2 \mid \mathrm{dead}>$. We need some more interpretive rules to make sense of this state.

The usual story goes as follows. The cat contains many particles, and where a particle ends up if the state is |alive $>$ is in general different from where it ends up in $\mid \mathrm{dead}>$. That is, the configuration of particles in a live cat is different from that in a dead cat, and differs for most of the particles. Further, the wavefunction only has significant amplitude in the regions of configuration space that correspond to these configurations. Therefore the state $1 / \sqrt{2} \mid$ alive $>+1 / \sqrt{2} \mid$ dead $>$ will have two lumps to it, one in the live cat configuration and one in the dead cat configuration. These lumps will be far from each other in many dimensions of configuration space. It is very, very unlikely that further Schrodinger evolution will ever bring the lumps together again. In technical terms, the state $1 / \sqrt{ } 2 \mid$ alive $>$ $+1 / \sqrt{ } 2 \mid$ dead $>$ decoheres.

Decoherence means that for all practical purposes one can think of there being two states, $1 / \sqrt{ } 2 \mid$ alive $>$ and $1 / \sqrt{ } 2 \mid$ dead $>$, each evolving independently of the other. But we still need to get from this to the existence of two cats, one alive 
and the other dead. That is, even granting that the physical situation is the sum of two pieces, we still need an argument the $1 / \sqrt{ } 2$ |alive $>$ piece represents a live cat and the $1 / \sqrt{ } 2 \mid$ dead $>$ piece a dead cat.

One issue is the factor of $1 / \sqrt{ } 2$. Why are we justified in ignoring this factor? The general answer to this is functionalism. However it is that a wavefunction like |alive > represents a physical situation with a live cat, we assume that all that is important are the relations of parts of the physical situation to one another. An overall scaling factor like $1 / \sqrt{ } 2$ will not change these relations, so $1 / \sqrt{ } 2$ alive $>$ represents a live cat just as much as $\mid$ alive $>$ does.

Notice: this answer relies on the premise that |alive > represents a live cat, but as of yet we have no account of how that comes about. |alive $>$ is a wavefunction with most of its amplitude concentrated in a region of the $3 \mathrm{~N}$-dimensional space over which it is defined, while |dead $>$ is a wavefunction concentrated in another region. Ignoring the labels "alive" and "dead", what makes the one a representation of a live cat and the other a representation of a dead cat?

One answer that is very tempting relies on the notion that the $3 \mathrm{~N}$-dimensional space is, indeed a configuration space, and that the clumping of the wavefunction in a particular region corresponds to $\mathrm{N}$ particles being arranged in the corresponding configuration. But in order to be entitled to this answer a theory must postulate the existence of $\mathrm{N}$ particles and a 3-dimensional space they exist in. This is something Many Worlds theorists refuse to do. And even postulating the particles and the space would not solve all the problems: the wavefunction clumps in regions of the $3 \mathrm{~N}$-dimensional space, not at a point, so there is no particular configuration that the wavefunction would indicate.

|alive $>$ has a particular mathematical form as a complex function of a $3 \mathrm{~N}$ - dimensional space. It is treated as a representation of a living cat. But it is not clear exactly how this representation is to be achieved. The mathematical object must be supplemented with a physical ontology and semantic rules specifying how the physical ontology is represented by the wavefunction. Let's consider three quite different approaches to this problem.

The first approach tries to take the wavefunction as nearly isomorphic to the physical ontology as possible. Since the wavefunction is a complex function on a $3 \mathrm{~N}$ - dimensional space, one postulates a $3 \mathrm{~N}$-dimensional physical space and a field, or pair of fields, on it. This position has been advocated by David Albert and has come to be known as wavefunction realism (Albert 1996, 277). ${ }^{1}$

Wavefunction realism makes the semantic rule connecting the wavefunction and the physical ontology easy. But the price it pays is making the connection between the physical ontology and the familiar world of experience hard. The world is not presented to us as in $3 \mathrm{~N}$-dimensional space but in 3-dimensional space. The familiar features of the cat, the features that distinguish the live cat from a dead cat, concern the three-dimensional structure of the cat and its parts. If the wavefunction represents physical reality this way, it is obscure how to recover that three-dimensional structure.

1 The terminology has been adopted in Ney and Albert 2013. See Wallace and Christopher Timpson 2010, 697. 
Albert's approach to this problem relies on functionalism. He seeks to show that the $3 \mathrm{n}$-dimensional situation is isomorphic to a 3 -dimensional one in the right way to produce an emergent 3-dimensional world. This requires an account of emergence, and in particular an emergence rather different from the way fluid behavior emerges from molecular interactions. The latter occurs because the collective action of the atoms produces behavior that is approximately that of a perfect fluid, at least at certain scales. Clearly getting a 3-dimensional space containing objects to emerge from a $3 \mathrm{~N}$-dimensional space is a different sort of relation.

Any successful physical theory must forge a connection between the physical world it posits and the familiar world of everyday experience. This approach to the wavefunction stands or falls on this point since the physical ontology does not obviously contain even approximations to the world of experience.

If one wants a 3-dimensional world in the physics the most obvious solution is to postulate it. Our second approach takes this tack, adding a real physical three-dimensional space to the ontology. But even with this in hand it is not immediately obvious how the wavefunction represents any material contents of the space. David Wallace and Chris Timpson have offered a suggestion called Spacetime State Realism. ${ }^{2}$ Mathematically, the question is how to define the material contents of space from the wavefunction.

There is a natural mathematical operation that can be applied to the wavefunction of a whole system to define a mathematical representation of a part of the system: taking the partial trace. Wallace and Timpson propose using this operation to assign properties to regions of 3-dimensional space, the properties being represented by the reduced density matrix that results from the operation.

By adding the 3-dimensional space and populating it we have managed to produce a physical ontology that could correspond to the world of experience. If dead > clumps the wavefunction in a region of configuration space corresponding to a dead cat then the properties will be $\mathrm{d}$ istributed in the corresponding way. Our state $1 / \sqrt{ } 2 \mid$ alive $>+1 / \sqrt{ } 2 \mid$ dead $>$ would have properties distributed in a live cat configuration and properties distributed in a dead cat configuration. And since the $1 / \sqrt{ } 2$ alive $>$ piece of the wavefunction evolves independently from the $1 / \sqrt{ } 2 \mid$ dead $>$ piece, the two cats will move independently of one another, passing through each other in the 3-space. We have a Many Worlds picture.

Mathematically the wavefunction in this Many Worlds theory is identical to the wavefunction of wavefunction realism; what has changed is the physical ontology and the semantic rule. We have gotten a 3-dimensional space containing an evolving set of properties because we postulated them in the physical ontology. So our first two approaches to understanding the wavefunction demonstrate that its mathematical form does not establish its ontological significance.

The Many Worlds approach, of course, has its own challenges. We have seen how it seems to get a multiplicity of cats from the Schrodinger evolution of the wavefunction, and how decoherence ensures that the cats will not molest each other. That same decoherence also ensures that the relative squared-amplitudes for the cats will be irrelevant to how they behave. That poses the question of why 
the relative squared-amplitudes should have any physical significance at all. But the squared-amplitude is taken to be the probability of the various outcomes, so if it has no physical significance then the Many Worlds theory will be powerless to explain the way the quantum-mechanical formalism is actually used.

There is another conceptual puzzle about Spacetime State Realism. It postulates a 3-dimensional world containing localized properties, but no particles. If there are no particles there are no configurations, so why is the space over which the wavefunction is defined treated as a configuration space? We have already seen that the mathematics of the wavefunction derived from classical configuration space, but if there are no particles that is a curious choice.

Our third approach starts with this aspect of the wavefunction. This approach postulates not just a 3-dimensional space but also $\mathrm{N}$ particles in the space. The wavefunction can now be interpreted as a complex function over the configuration space of the system.

Once a theory postulates particles, the essential question is how the particles move. The motion of the particles constitutes a change in the configuration of the particles, so the evolution of the system corresponds to a trajectory through configuration space. Since the wavefunction can now be interpreted as a complex function on the configuration space of the system, it is natural to ask whether the trajectory could be determined by the wavefunction. Indeed it can, and the theory postulating the simplest rule here is the pilot wave theory or Bohmian Mechanics. The way the wavefunction determines the evolution of the configuration is specified by the guidance equation.

Bohmian mechanics has a physical ontology with three parts: a 3-dimensional space, $\mathrm{N}$ particles in the space, and a physical item represented by the wavefunction. Let's call the item represented by the wavefunction the quantum state of the system. What is the semantic rule connecting the wavefunction and the quantum state?

Mathematically different wavefunctions represent the same quantum state. If the wavefunction is changed by an overall phase the physical condition it represents doesn't change because the trajectories it produces don't change. Rescaling the magnitude uniformly also makes no difference to the motions produced by the guidance equation. In technical terms, the quantum state corresponds not to a vector in the Hilbert space of square-integrable complex functions over configuration space, but rather a ray. What most directly corresponds to the quantum state, in other words, is an element of projective Hilbert space.

Notice the dialectic between the mathematical representation and the physical ontology. At times the mathematical representation suggests a physical item, as with Spacetime State Realism, while at other times the physical ontology suggests adjustments to the mathematical apparatus or semantic rule. This is why the purely mathematical aspects of the theory can be uncontroversial but the ontological implications still remain obscure.

In the Bohmian theory the wavefunction represents the quantum state, which plays a purely dynamical role, guiding the particles. In Spacetime State 
Realism the wavefunction plays a dual role, representing a quantum state and also a property distribution in 3-dimensional space. In wavefunction realism the wavefunction represents a real physical field on a $3 \mathrm{~N}$-dimensional space.

The difference in ontologies necessitates differences in the semantic rules. While a change in the overall phase of the wavefunction does not change the quantum state represented in Bohmian mechanics it does imply a different physical situation in wavefunction realism. The change in phase will not affect the property distribution in Spacetime State Realism, but it is open whether the quantum state would change. Presumably the intention is that the quantum state represented also remains the same.

The wavefunction does double duty in Spacetime State Realism because there is a dual ontology - a quantum state on the one hand and a property distribution in 3- dimensional space on the other-but only a single mathematical object. Bohmian mechanics also has a dual ontology but it employs dual mathematical representations: a point in configuration space to represent the disposition of the particles and a field on configuration space to represent the quantum state. The duality appears also in the dynamics, with the Schrodinger equation determining how the quantum state evolves and the guidance equation how the particles move.

The dualism in Bohmian mechanics has often been used as a point of criticism: the theory is said not to be quantum mechanics because it adds something - the particles-to the ontology. Many Worlds theory, in contrast, has been presented as an ontologically monistic theory. But this is misleading: Spacetime State Realism is a dualistic ontology that happens to use the same mathematical object to represent both parts of the ontology.

Not every advocate of Many Worlds is a Spacetime State Realist. But a monistic Many Worldser only has the quantum state to work with, and it does not exist in 3- dimensional space. The question of how to connect the ontology of the theory to experience therefore becomes acute. Since the evidence for the theory is expressed in terms of happenings in 3-dimensional space there must be something in the ontology of the theory that corresponds to such happenings.

There are, of course, other issues that the Many Worlds theory must face, particularly the understanding of probability in the theory. But those questions cannot even be properly framed before the physical ontology and semantic rule for interpreting the wavefunction are given.

Why hasn't this problem been more widely acknowledged? To a great extent, it has been disguised by a linguistic trick. For example, we discussed the Schrodinger cat problem using the wavefunctions called |alive $>$ and $\mid$ dead $>$. It is all too easy to slip into thinking that |alive> represents a physical state containing a live cat and $\mid$ dead $>$ represents a state containing a dead cat just because of how they have been labeled. But a wavefunction has to earn the right to the label via the physical ontology that it represents. Without representing some matter in a 3-dimensional space it is unclear how a wavefunction could indicate a cat in any state. 
How does Bohmian mechanics do on this score? In that theory the wavefunction does not represent any matter in 3-dimensional space. But the dynamics of the theory implies that the actual configuration of the particles will typically inhabit a location in configuration space where the wavefunction has a high magnitude. So a wavefunction like |alive>, which is non-zero only in regions of configuration space that correspond to a configuration of a live cat, implies that the actual configuration is really in one of those states. The wavefunction does not directly represent the matter distribution, but it has implications for the matter distribution.

Let's look at one last issue: the collapse of the wavefunction. Standard presentations of quantum theory postulate that the wavefunction collapsesundergoes a sudden radical change-when a measurement occurs. This formulation runs afoul of the measurement problem since it is not clear exactly what physical conditions are required for a measurement to occur. How do our theories deal with this?

As we mentioned, quantum Bayesianism has a clear account of collapse. Collapses occur when one receives new information about the system, and are to be expected if the wavefunction represents information. The Many Worlds theory rejects the collapse-the wavefunction always obeys Schrodinger's equationand reaps the consequence that there are many worlds after a measurement-type interaction. But what about Bohmian mechanics?

The wavefunction never collapses in that theory. But this does not yield many cats in a Schrodinger cat situation because the cat is composed of particles and the particles are in a definite configuration at all times. There is an additional fact: the particles are only influenced by the quantum state where the actual configuration is. In a Schrodinger cat situation the quantum state after the experiment will be the uncollapsed state represented by $1 / \sqrt{ } 2 \mid$ alive $>+$ $1 / \sqrt{2} \mid$ dead $>$. The actual configuration of the cat will either be in the region of configuration space associated with |alive $>$ or that associated with $\mid$ dead $>$. But since those two states will have decohered, one can safely throw away the piece that does not correspond to the actual configuration. In this way there is an effective collapse of the wavefunction even though there is no physical collapse.

The effective collapse again illustrates the dialectic between the physical ontology and the mathematical representation. Because it is part of everyday practice to collapse the wavefunction one might well expect the dynamics of the theory to have a collapse in it. But the Bohmian theory does not postulate any sudden change in the physical dynamics. Rather, collapsing as a mathematical step is justified by the character of the dynamical laws.

There is no royal road from the mathematical formalism to the physical ontology. It should be the part of the physicist to make clear what the physical ontology is and how it is represented. But at least in the case of quantum theory mainstream physics has not articulated a clear theory. Work in the foundations of physics-work done by physicists, mathematicians and philosophers-seeks to fill these gaps. 
There is a certain amount of free play between the mathematical representation and the physical ontology. We have seen how this allows for competing physical accounts using the same mathematical formalism. There are questions of which parts of the mathematical formalism represent physical ontology. And there are further questions about the relation between the physical ontology and the world as we experience it. It is here, between the mathematics and the physics and the lived world, that philosophical work can thrive, both clearly articulating the ontologies of existing theories and investigating novel possibilities.

\section{References}

Albert, David. 1996. "Elementary Quantum Metaphysics." In Bohmian Mechnaics and Quantum Theory: An Appraisal, edited by James Cushing, Arthur Fine and Sheldon Goldstein, 277-284. Dordrecht: Kluwer.

Fuchs, Christopher A., N. David Mermin, and Rudiger Schack. 2014. "An Introduction to QBism with an Application to the Locality of Quantum Mechanics." American Journal of Physics 82(8): 749-754. doi: 10.1119/1.4874855

Ney, Alissa, and David Albert, eds. 2013. The Wave Function. Oxford: Oxford University Press.

Wallace, David, and Christopher Timpson. 2010. "Quantum Mechanics on Spacetime 1: Spacetime State Realism." British Journal for the Philosophy of Science 61(4): 697-727. doi: 10.2307/40981311Dae net es mi, cum earchicabori blabo. Itaquib usandebitia voluptatur magnis nectur simendis deles pratemporem qui ommos autem inctur, quibus nobit occaero restemquid eat. 